\title{
TRANSCRIPTOME SEQUENCING ANALYSIS OF GOAT TESTICULAR TISSUE
}

\author{
Q. HE, Z. ZHAO*, Z. DAI, L. QIAO AND T. YANG \\ Chongqing Engineering Research Center for Herbivores Resource Protection and Utilisation \\ Southwest University, Chongqing- 400 716, China
}

Testicular development and spermatogenesis play vital roles in goat reproduction and production. However, only a minimal quantity of genomic information is available on goat testicles during the embryonic stage, adolescence and adulthood. In this study, the transcriptomes of Dazu Black goat testis from infancy (ET), puberty (PT) and adulthood (AT) were sequenced using RNA-Seq technology with Illumina Hiseq4000, and comparative analyses of transcriptomes between three life stages (pairwise comparison) were performed. The sequencing results showed that a total of 62,905 transcriptionally expressed genes were detected, of which 12,829 were new and 42,268 were known. There were extensive alterations in gene expression in the testis over the course of maturation. The gene expression pattern in ET was distinct from those in $\mathrm{AT}$ and $\mathrm{PT}$, which indicates that spermatogenesis may be initiated after PT. We analyzed a large number of genes related to spermatogenesis and testicular development, which play important roles at different developmental stages. We also found that the MAPK, tight junction and Wnt signaling pathways were significantly involved at different developmental stages. The results of this study identified differences in mRNA expression in goat testis over the course of its development and the information will be useful for future studies of spermatogenesis in Dazu Black goats.

Key words: Spermatogenesis, Testis, Transcriptome

In China, goats (Capra hircus) are important farm animals, providing meat, milk, fiber and fur (Zhu et al., 2013). As an important economic meat animal in China, goat has the characteristics of black hair and high reproductive capacity (Zhao et al., 2015). The testes in male mammals regulate sexual function, secrete sex hormones and produce spermatozoa, and testicular development directly affects the reproductive performance of bucks. In recent years, an increasing number of studies on goat testicles (Sharma et al., 2016; Szatkowska et al., 2017) have involved the study of mRNA (Samir et al., 2015), testicular development and

*Corresponding Author 
spermatogenesis (Barcelos et al., 2016). The function of the testis is regulated by the hypothalamic-pituitary-gonadal axis, and the expression of genes and hormones in each stage stimulates the development of the testis and the production of testosterone (Archana et al., 2014). Previous studies (Lejeune et al., 1998) have shown that testicular tissue of 0 day age was in an undifferentiated state, and the seminiferous tubules were small, only containing spermatogenic stem cells (SSCs) and Sertoli cells. At the age of 2 months, the cell composition of the testicles have not changed significantly, but spermatogenic stem cells have begun to differentiate. Testicular tissue develops gradually, testicular development is complete by 6-8 months of age, and many kinds of cells are present in mature testes, including sperm cells (Faucette et al., 2014). Therefore, in this study, we selected testicular tissues from 3 age groups of Dazu Black goats, namely, 1 day, 2 months and 9 months, and we used paraffin tissue section observation to confirm that the testicles of the three age groups collected were at the appropriate growth and development stage. Significant mRNA related to testicular development and reproductive regulation has been found in previous studies (Dere et al., 2017; Guan et al., 2017; Jin et al., 2015); however, the database related to the growth and development of goat testicular tissue is not abundant. For this purpose, we will use transcriptome sequencing technology to analyze and identify mRNA of testicular tissues in infancy, adolescence and adulthood.

Thus, the purpose of this study was to use transcriptome sequencing to detect the expression of differentially expressed genes in three stages of testicular tissue. The selection of testicular tissue rather than single-cell sequencing is intended to provide more extensive access to genetic data related to the development of testes, spermatogenesis and cell maturation. The differentially expressed genes (DEGs) were classified by sequencing, and their biological functions were studied. In this study, we studied the structural characteristics of goat testicles in three age stages through immune observation.

\section{MATERIALS AND METHODS} Experimental animals and sample collection: The male Dazu Black goats in the experiment were cared for by the Herbivores Institute at Southwest University. Dazu Black goats reach puberty at 2-3 months of age, and at 6-8 months, they show mature fertility and are sexually mature. In this study, healthy goats at birth without obvious reproductive abnormalities (normal testicular development, no obvious reproductive health problems) were selected and reared in the same environment and under the same feeding conditions and were kept healthy throughout the study. Before choosing a goat, both testes in the scrotum were confirmed by manual 
palpation. Goats ingested concentrated feed, hay and water. The testicular collection experiments were approved by the Committee on the Ethics of Animal Experiments of Southwest University (No. [2007] 3) and the Animal Protection Law in China, and all efforts were made to minimize suffering.

\section{Sample collection and total RNA} extraction: In this experiment, testicular tissues from Dazu Black goats (three goats at each stage) at infancy (aged one day, ET), preadolescence ( 2 months old, PT) and adulthood (9 months old, AT) were collected. Tissues were then washed with PBS buffer and alcohol on the surface and attachments, and one side of the testis was placed in formaldehyde solution, paraffinized, sectioned, and stained with HE (hematoxylin-eosin staining); the other side of the testicle was cryopreserved in dry ice and transported to the laboratory to extract total RNA. Total RNA was extracted from the 9 tissues using TRIzol reagent (Invitrogen, Carlsbad, CA, USA) following the manufacturer's instructions (Connolly et al., 2006). The sample was mixed with fragmentation buffer (Ambion, Carlsbad, CA, USA) for RNase-free DNase I treatment. The concentration and quality of total RNA was determined and quantified by a QUBIT RNA ASSAY KIT and a 2100 Bioanalyzer (Agilent Technologies, Santa Clara, CA, USA).

Library construction and sequencing: Sequencing was performed with the
Illumina sequencing platform (Illumina HiSeq $^{\text {TM }}$ 4000) using single-end paired-end technology in a single run by The Beijing Genomics Institute (BGI)-Shenzhen, Shenzhen, China. All raw reads were removed, and the reads with a high $\mathrm{N}$ content (over 3\%) and low quality (when the full length of a read was less than $50 \mathrm{bp}$ or when over $50 \%$ of the bases in a read had a quality value of $\leq 3$ ) were filtered. Then, we used HISAT (Kim et al., 2015) to compare clean reads to the reference genome and then assembled the reads with String Tie (Pertea et al., 2015). We used BLAST (Altschul et al., 1990) or Diamond (Buchfink et al., 2015) to carry out NT (nucleotide collection database), NR (nonredundant protein database), COG (clusters of orthologous groups), KEGG (https://www.genome.jp/kegg/) and SwissProt annotation of mRNA, and we used Blast2GO (Conesa et al., 2005) (http:/ /www.geneontology.org/) and NR annotation results for gene ontology database (GO) annotation. Then, we proceeded with the deep analyses, including differential gene expression, mRNA annotation, insertion-deletion (InDel), and single nucleotide polymorphism (SNP) analysis.

Bioinformatic analyses: In this experiment, three biological replicates were used, and the Pearson correlation coefficient was squared $\left(\mathrm{R}^{2} \geq 0.092\right)$. To identify genes showing a significant change in expression at the three different time 
points, we used the difference analysis software DEGseq to analyze the differences between groups. Differentially expressed genes were estimated from the number of fragments per kilobase per million mapped reads (FPKM). To quantify expression, the coverage of each transcript was computed by applying Bowtie2 (Langmead and Salzberg, 2012). Based on this method, it is possible to evaluate probability counts that are more or less frequent between groups. All transcripts that were differentially expressed between the two groups qualified for annotation. The resulting P-values were fit, and the threshold for significantly differential expression was selected as the difference multiples $\geq 2$ and FDR $\leq 0.001$.

\section{RESULTS}

\section{Observation of testicular tissue (paraffin} section): The histomorphological developmental status of the testes is shown in Fig. 1. This study included nine goat testes in three age groups. In the infancy period (aged one day), the testicles were found to be undifferentiated and to contain only spermatagonial stem cells and Sertoli cells; the seminiferous tubules were small, and there were 1-2 Sertoli cells per tubule. In early adolescence ( 2 months), the testicles were found at the transitional stage; existing spermatagonial stem cells had begun to differentiate into spermatocytes but did not exhibit sperm cell production. When goats reached adulthood (9 months), testicular development was complete, the seminiferous tubule was enlarged, the cell type was increased, and mature sperm cells appeared. The Sertoli cells were in the mature stage (sc) and were larger than SSCs (de Rooij., 2001; Saitou and Yamaji, 2012).

\section{Extraction and identification of RNA:}

The quality of total RNA extracted from testicular tissue was detected by an Agilent 2100 instrument and screening out the samples that met the detection standard of BGI gene sequencing samples (RNA sequencing sample quality standards) for sequencing purposes. The RNA quality test results are shown in Fig. 2, indicating that all test samples meet the requirements of library construction sequencing, and most samples can meet the requirements of library construction two or more times.

RT-PCR assay sequencing results: To test the accuracy of the results of transcriptome sequencing gene expression data, we randomly selected four significant DEGs for verification to perform real time quantitative PCR (qPCR). Each trial was repeated three times to increase the reliability of RNA-seq results. The results of real-time quantitative polymerase chain reaction ( $\mathrm{RT}-\mathrm{PCR}$ ) showed that the expression trend of the two results was consistent (Fig. 3). This indicated that the results were highly credible. Transcriptome sequencing of these genes in the testicles of goats at three different stages of development is illustrated. 

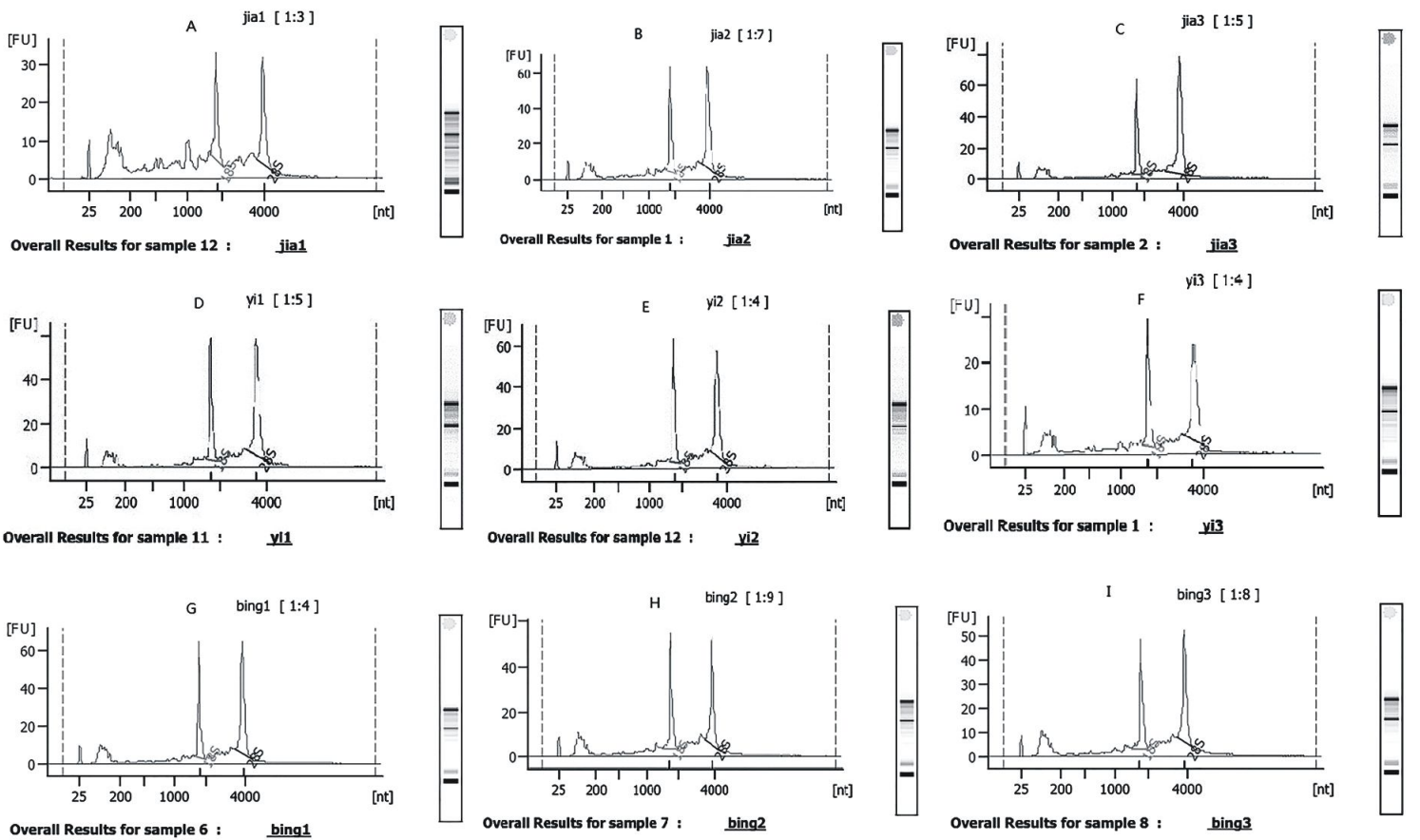

[A-C is the test report of three samples of AT, D-F is the test report of three samples of PT and G-I is the test report of three samples of ET. The bar on the right of each graph is the simulated electrophoresis graph.]

Fig. 2. Agilent2100 detection peak diagram

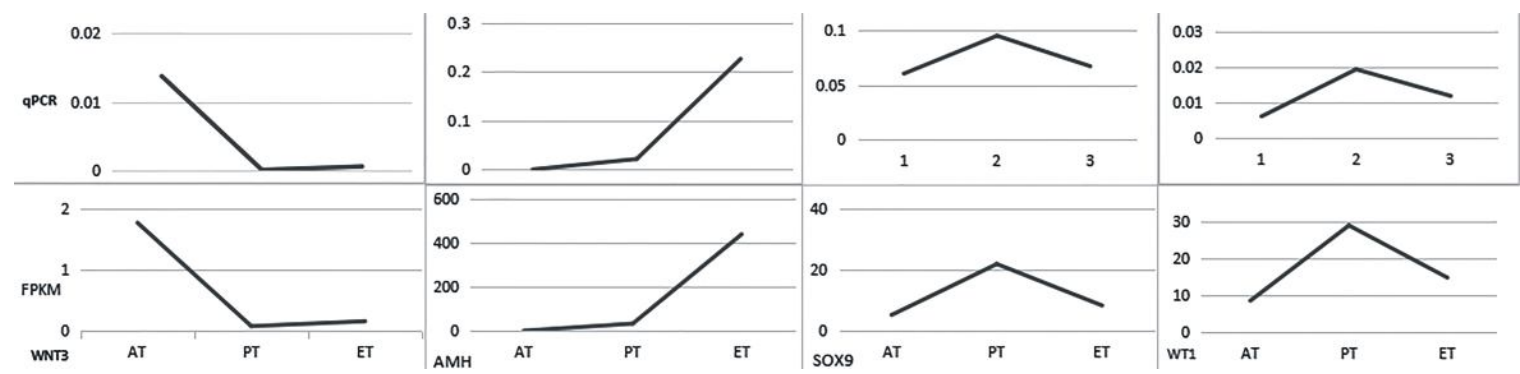

[The accuracy of sequencing results was verified by qPCR experiment. Broken line graph shows the expression trend of genes in three age groups. The top figure displays the qPCR results, and the bottom figure exhibits the FPKM values of genes in the sequencing results.]

Fig. 3. Gene expression trend map 


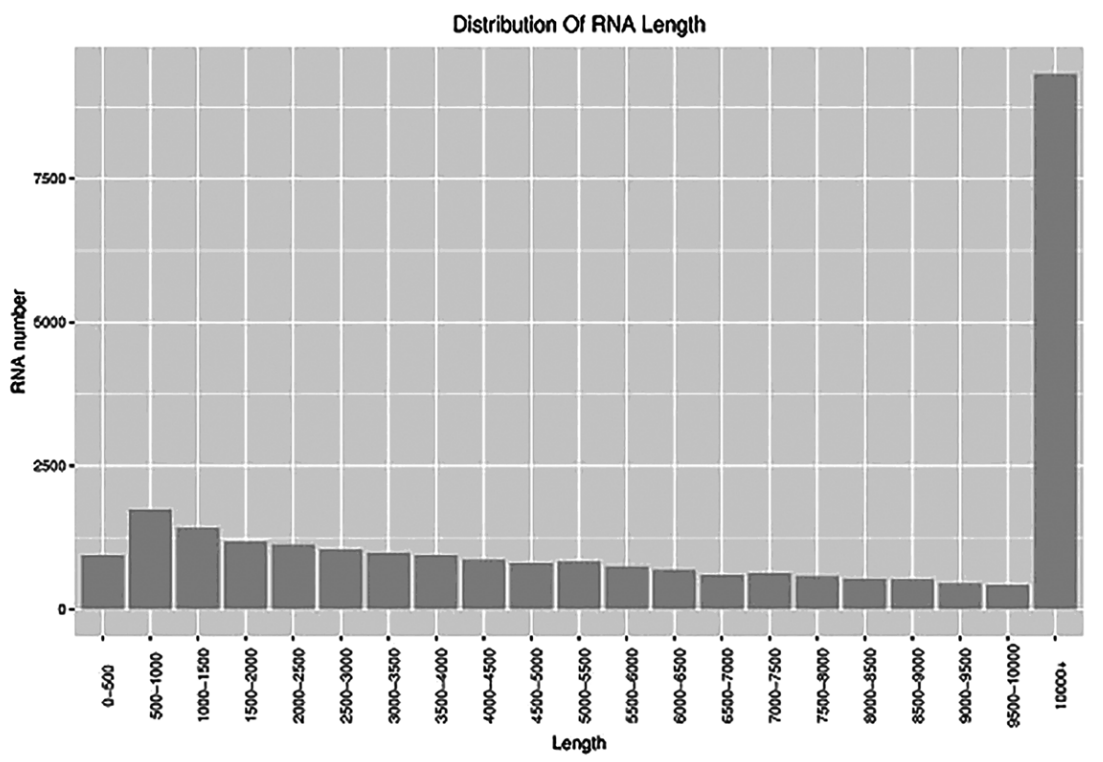

Fig. 4. The length distribution of the transcripts
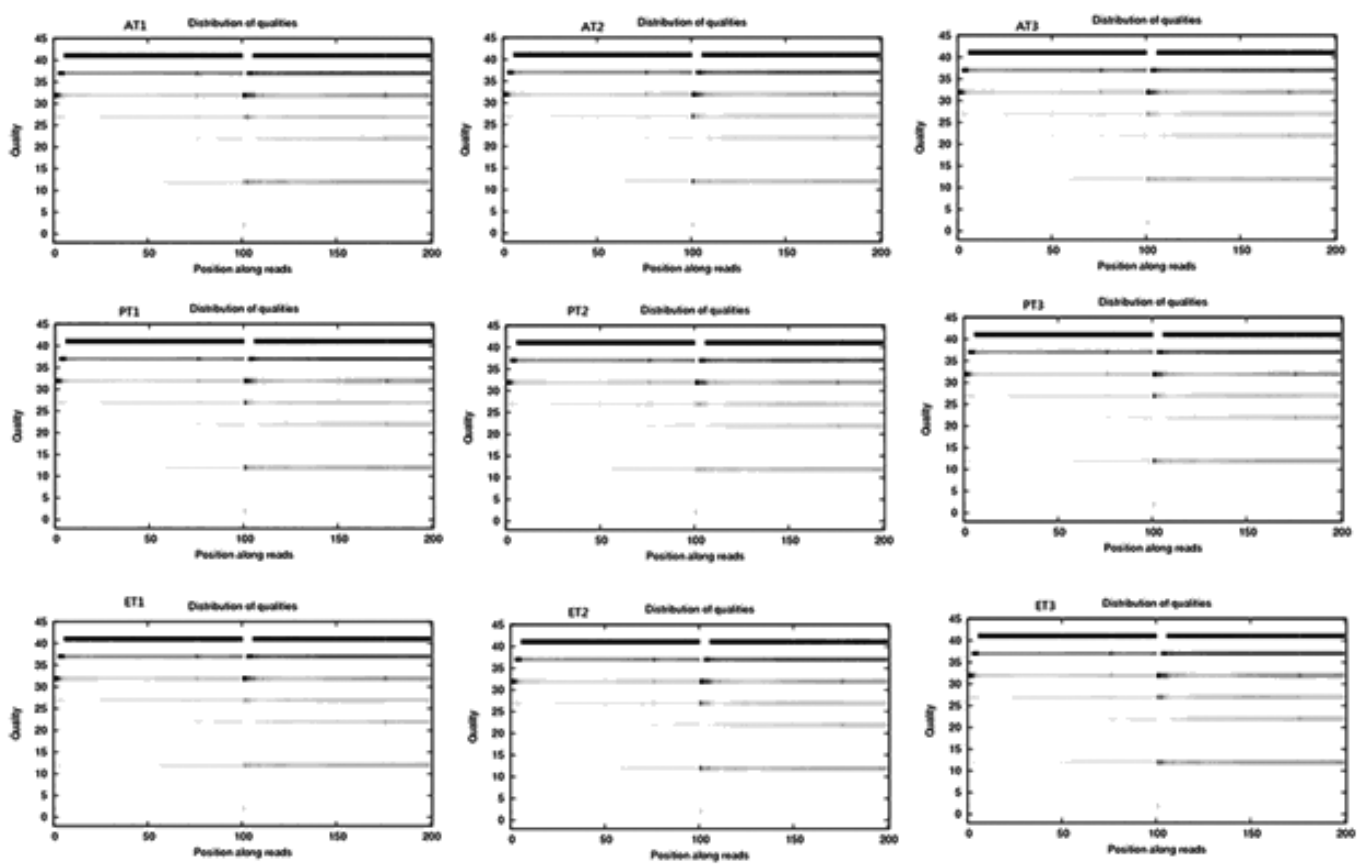

Fig. 5. The mass distribution of the bases (AT1-ET3): $\mathrm{X}$ axis- base position read; $\mathrm{Y}$ axisthe quality of the base 
Illumina sequencing and de novo assembly: A total of 9 goat samples were measured using the Illumina HiSeq platform, and the average output of each sample was $12.68 \mathrm{~Gb}$. The average ratio of the genome was $91.39 \%$. A total of 62,905 transcripts were detected, of which 12,829 were new transcripts and 42,268 were known transcripts. The length distribution of the transcripts is shown in Fig. 4. Table 1 shows the summary of the
Illumina assembly and analysis of transcriptomic sequences in the testicular samples of 9 Dazu Black goats. Goat testes transcriptome libraries were constructed from the infancy, early adolescence and adult stages. The results showed that the total clean reads ratio was over $90 \%$, with an average of 95\%. The Q20 and Q30 of each transcriptome library were above $99 \%$ and $97 \%$, respectively, indicating that all libraries were of good quality (Fig. 5).

Table 1. Summary of reads and matches

\begin{tabular}{lcccccc}
\hline & $\begin{array}{c}\text { Total Clean } \\
\text { Base }\end{array}$ & $\begin{array}{c}\text { Total Clean } \\
\text { Reads Ratio }\end{array}$ & $\begin{array}{c}\text { Read with NRate } \\
\text { Exceed Ratio }\end{array}$ & $\begin{array}{c}\text { GC } \\
\text { of Fq1 }\end{array}$ & $\begin{array}{c}\text { Q20 } \\
\text { of Fq1 }\end{array}$ & $\begin{array}{c}\text { Q30 } \\
\text { of Fq1 }\end{array}$ \\
\hline AT1 & 12587643000 & $96.09 \%$ & $0.01 \%$ & $46.27 \%$ & $99.26 \%$ & $97.66 \%$ \\
AT2 & 12711402200 & $94.86 \%$ & $0.01 \%$ & $46.71 \%$ & $99.29 \%$ & $97.74 \%$ \\
AT3 & 12715733600 & $95.84 \%$ & $0.01 \%$ & $48.02 \%$ & $99.28 \%$ & $97.68 \%$ \\
PT1 & 12719075000 & $93.86 \%$ & $0.01 \%$ & $47.70 \%$ & $99.25 \%$ & $97.61 \%$ \\
PT2 & 12706113600 & $95.65 \%$ & $0.01 \%$ & $46.26 \%$ & $99.27 \%$ & $97.68 \%$ \\
PT3 & 12676244400 & $94.44 \%$ & $0.01 \%$ & $45.68 \%$ & $99.22 \%$ & $97.56 \%$ \\
ET1 & 12605944000 & $91.45 \%$ & $0.01 \%$ & $46.77 \%$ & $99.26 \%$ & $97.66 \%$ \\
ET2 & 12691315600 & $94.29 \%$ & $0.01 \%$ & $45.64 \%$ & $99.24 \%$ & $97.62 \%$ \\
ET3 & 12715358400 & $96.86 \%$ & $0.01 \%$ & $45.53 \%$ & $99.27 \%$ & $97.68 \%$ \\
\hline AT
\end{tabular}

AT1-3: adult testicular tissues 1-3 at month 9; PT1-3: preadolescence goat testes tissues 1-3 at month 2; ET13: infancy goat testes tissues 1-3 at day 1. Q20: the probability of an incorrect base call 1 in 100 reads; Q30: the probability of an incorrect base call 1 in 1,000 reads

Table 2. RNA quality test results

\begin{tabular}{lcccccccc}
\hline Sample & $\begin{array}{c}\text { Serial } \\
\text { number }\end{array}$ & $\begin{array}{c}\text { Group } \\
\text { number }\end{array}$ & $\begin{array}{c}\text { Amount Concentration Volume } \\
(\mathbf{n g} / \mu \mathbf{L})\end{array}$ & $\begin{array}{c}\text { Total } \\
\text { content }(\mu \mathbf{g})\end{array}$ & RIN & $\begin{array}{c}\mathbf{2 8 S} / \\
\mathbf{1 8 S}\end{array}$ \\
\hline AT1 & jia1 & TWH1707009423 & 1 & 624 & 20 & 12.48 & 6.5 & 1.1 \\
AT2 & jia2 & TWH1707009424 & 1 & 1808 & 20 & 36.16 & 8.0 & 1.4 \\
AT3 & jia3 & TWH1707009425 & 1 & 1176 & 60 & 70.56 & 9.1 & 2.1 \\
PT1 & yi1 & TWH1708005748 & 1 & 984 & 100 & 98.4 & 8.9 & 1.6 \\
PT2 & yi2 & TWH1708005749 & 1 & 835 & 100 & 83.5 & 8.6 & 1.4 \\
PT3 & yi3 & TWH1708005750 & 1 & 645 & 100 & 64.5 & 8.2 & 1.4 \\
ET1 & bing1 & TWH1707009429 & 1 & 940 & 20 & 18.8 & 8.3 & 1.4 \\
ET2 & bing2 & TWH1707009430 & 1 & 1840 & 20 & 36.8 & 8.1 & 1.1 \\
ET3 & bing3 & TWH1707009431 & 1 & 1692 & 60 & 101.52 & 8.3 & 1.2 \\
\hline
\end{tabular}

RNA Integrity Number (RIN) reflects the RNA integrity of samples. The closer the value is to 10, the higher the RNA integrity, whereas the smaller the RIN value is, the worse the integrity. 28S/18S is another indicator to evaluate the RNA integrity of samples 
Identification of differentially expressed genes: In transcriptome sequencing analysis, we estimated the number of genes that showed significant changes at different stages of development based on the number of FPKM (fragments per kilobase per million mapped reads). In this study, hierarchical clustering analysis was conducted for the genes expressed in 3 libraries (Fig. 6). Goat testicles at ET and PT showed similar gene expression profiles; the AT gene expression profiles showed a comparatively large difference from the other two groups. This suggests that the biological processes of the AT goat testicle and the ET and PT goat testicles have obvious differences and that the primary difference lies in the fact that there is sperm production in the testis of AT.

The read count data obtained from the transcriptome were used to analyze differences in gene expression; we determined the expression levels of all genes between AT-VS-PT, PT-VS-ET and AT-VS-ET in our RNA-Seq data based on gene expression profiles (Fig. 7). In terms of gene expression level analysis, we found that only LOC100860923 had an FPKM value greater than 10,000 in the AT group. There were 6 genes with values greater than 1,000: HMGB4,FAM229B, UBB, LOC102189 079, HSPB9, and TNP1, which were significantly higher in AT than the other two stages, but only UBB was highly expressed in the three tissues. For gene expression analysis in ET, we found that there were no genes with an FPKM value of more than 10,000 in $\mathrm{ET}$, but 51 genes with an FPKM value of more than 1,000, mainly RP (ribosomal protein) genes: RPL6, RPL12, RPL6, RPL7A, RPS23, RPL11, RPS27, RPS10, RPL5, COCIA1, COC1A2, TPT1, SPARC, RACK1, APOA1 GSTM3, EEF1A1 etc. Among them, except COCIA1 and COC1A2 are only highly expressed in ET, the rest genes were highly expressed in the three groups.

The resulting P-values were fit, and the threshold for significantly differential expression was selected as a corrected Pvalue of 0.05 and $\log 2$ (fold change). The results indicated a large number of differentially expressed mRNA between the three groups. We obtained 14,724 differentially expressed mRNA, including 7,012 upregulated mRNA and 7,712 downregulated mRNA, in AT-VS-ET. Moreover, 13,108 differentially expressed mRNA, including 6,029 upregulated mRNA and 7,079 downregulated mRNA, were identified in PT-VS-ET. A total of 6,021 differentially expressed mRNA, including 3,176 upregulated mRNA and 2,845 downregulated mRNA, were identified in PT-VS-ET (Fig. 8).

A Venn diagram showing the number of differentially expressed mRNA among the three pairwise comparisons is shown in Fig.9. Thus, a total of 16,051 genes were identified in the three transcriptome libraries, including 14,306 in AT, 13,230 in PT and 13,392 in ET (Fig. 9A). Fig. 9B shows the VN diagram of the differentially expressed genes. There were 1,704 
Indian Journal of Animal Health, June, 2019

Transcriptome sequencing analysis of goat testicular tissue
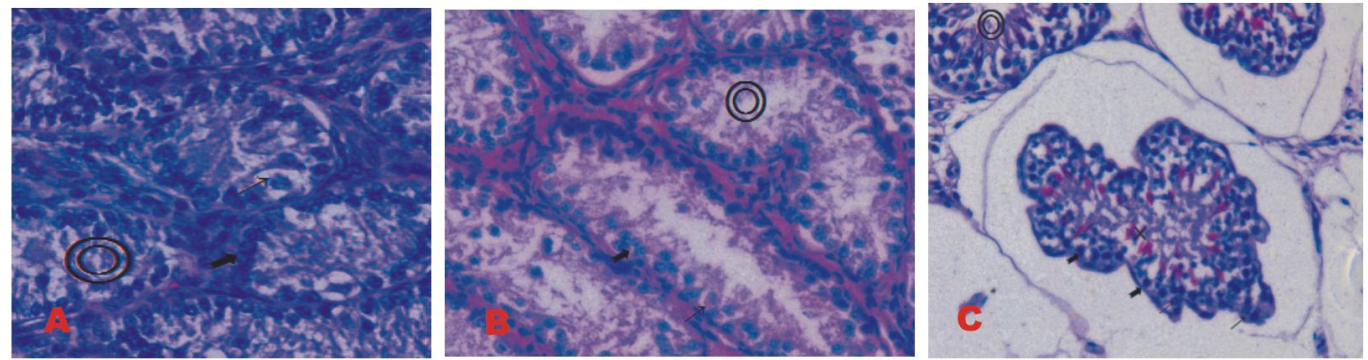

[Immunocytochemical (A to C) evaluations of goat testis in 3 developmental stages using hematoxylin and eosin (HE) staining. A: Spermatogonial stem cells (coarse arrow), Sertoli cells (arrow) and Seminiferous tubules (circle) during the infancy period. B: In early adolescence (2 months). C: In adulthood, sperm cells are present (x).]

Fig. 1. Immunohistochemical identification of goat testis

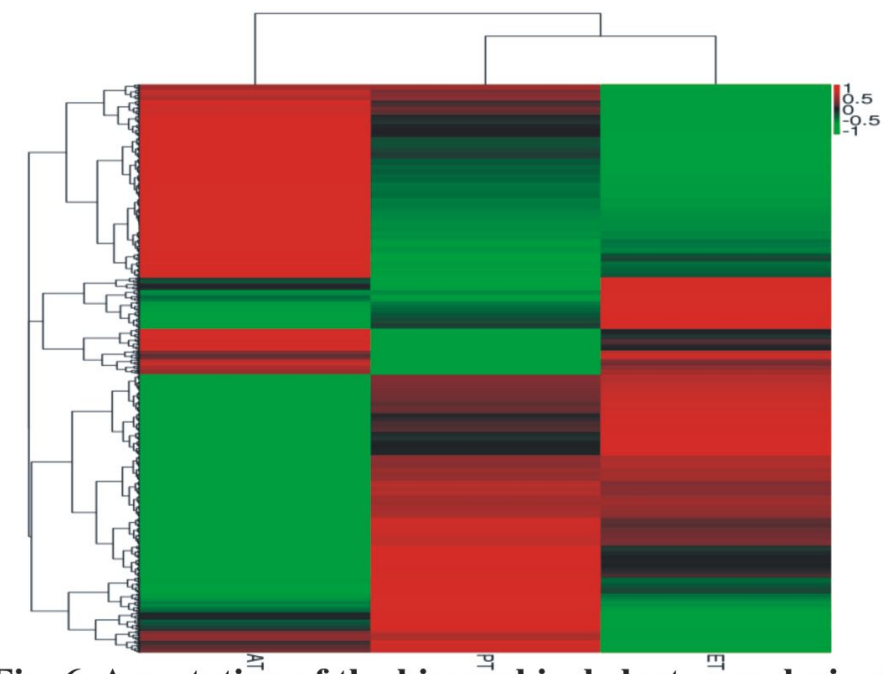

Fig. 6. Annotation of the hierarchical cluster analysis of genes
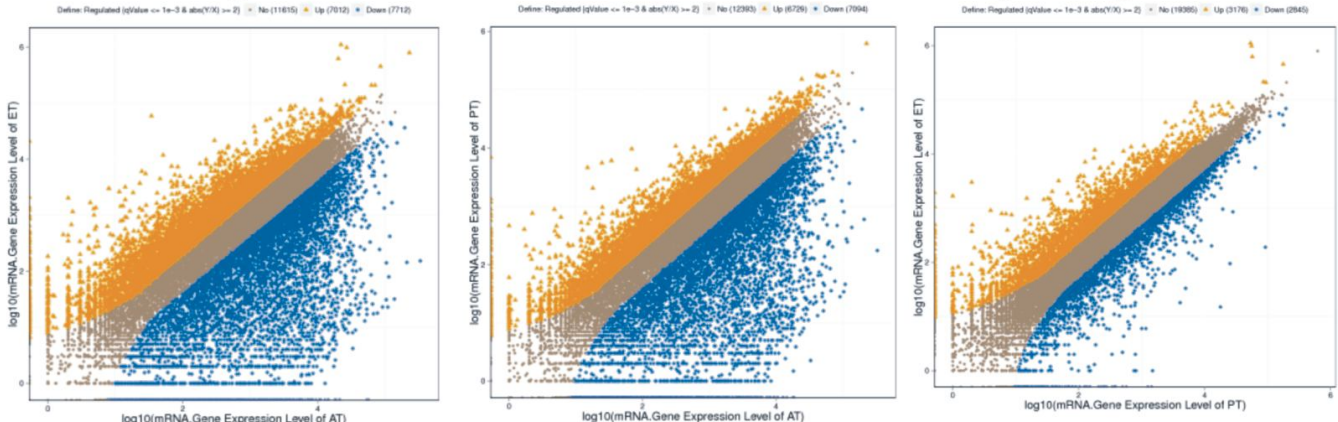

[Yellow dots represent upregulated genes, blue dots downregulated genes, and gray dots represent equally expressed genes.]

Fig. 8 Scatter plot of differentially expressed genes in the three libraries 
differentially expressed genes in AT-VSPT, 2,935 in AT-VS-ET and 551 in PTVS-ET.

\section{Annotation and functional classification:} Gene ontology (GO) is an international standardized gene function classification system that is used to comprehensively describe the genetic characteristics and products of organisms. All putative protein sequences of the Dazu Black goat were assigned for GO terms based on sequence functional similarity, and then Go Pipe software was used to perform GO functional classifications. GO analysis based on biological process enrichment was performed for sets of DEGs with significant cluster profiles. Only significant GO categories with $\mathrm{P}$-values $<0.05$ were chosen for analysis. The results of the GO enrichment analysis of DEGs (isoforms) were classified into three categories: BP, cellular component (CC) and molecular function (MF). Fig. 10 shows the GO classifications of the DEGs from the comparisons between AT and PT, PT and $\mathrm{ET}$, and $\mathrm{AT}$ and ET.

The unigenes of three stages of testicular tissue were assigned to three major GO categories and 64 subcategories. Of these, 89,393 in AT-VS-ET comprised the largest category, biological process, followed by cellular component $(62,870)$ and molecular function $(19,869)$. The other two groups shared the same trend. Among the molecular functions category, the top five results were involved in binding, catalytic activity, transporter activity, molecular transducer activity and nucleic acid binding transcription factor activity. Regarding cellular components, cell and cell part were the dominant groups; next were organelle, membrane and organelle part. Within the biological processes category, cellular process was the most dominant group, next were single-organism process, metabolic process, biological regulation and regulation of biological process.

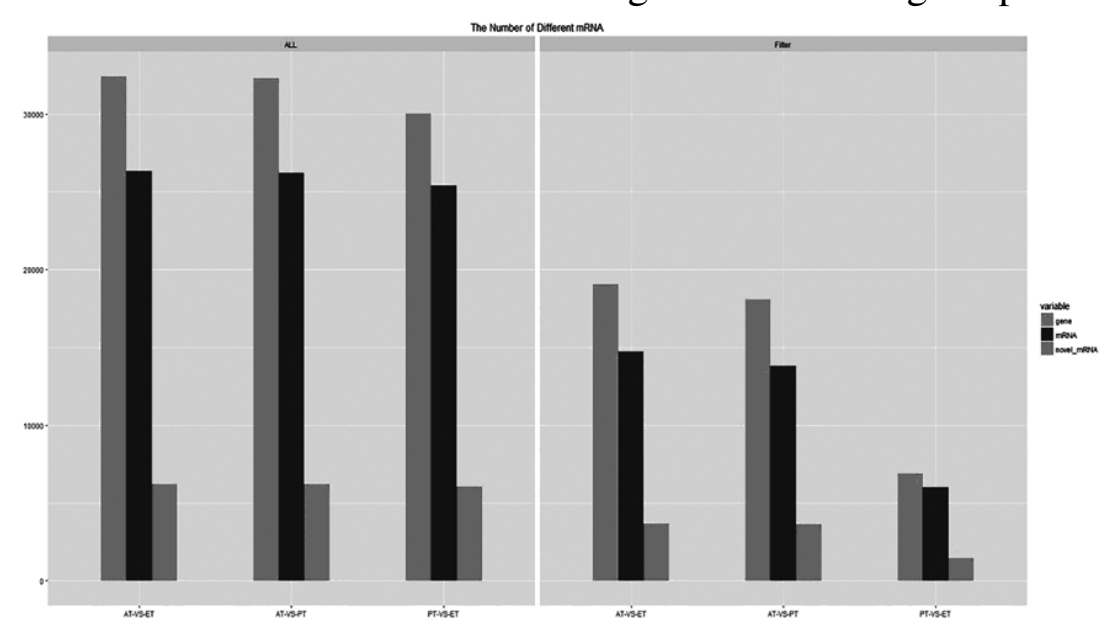

Fig. 7. Differentially expressed genes in the three libraries 


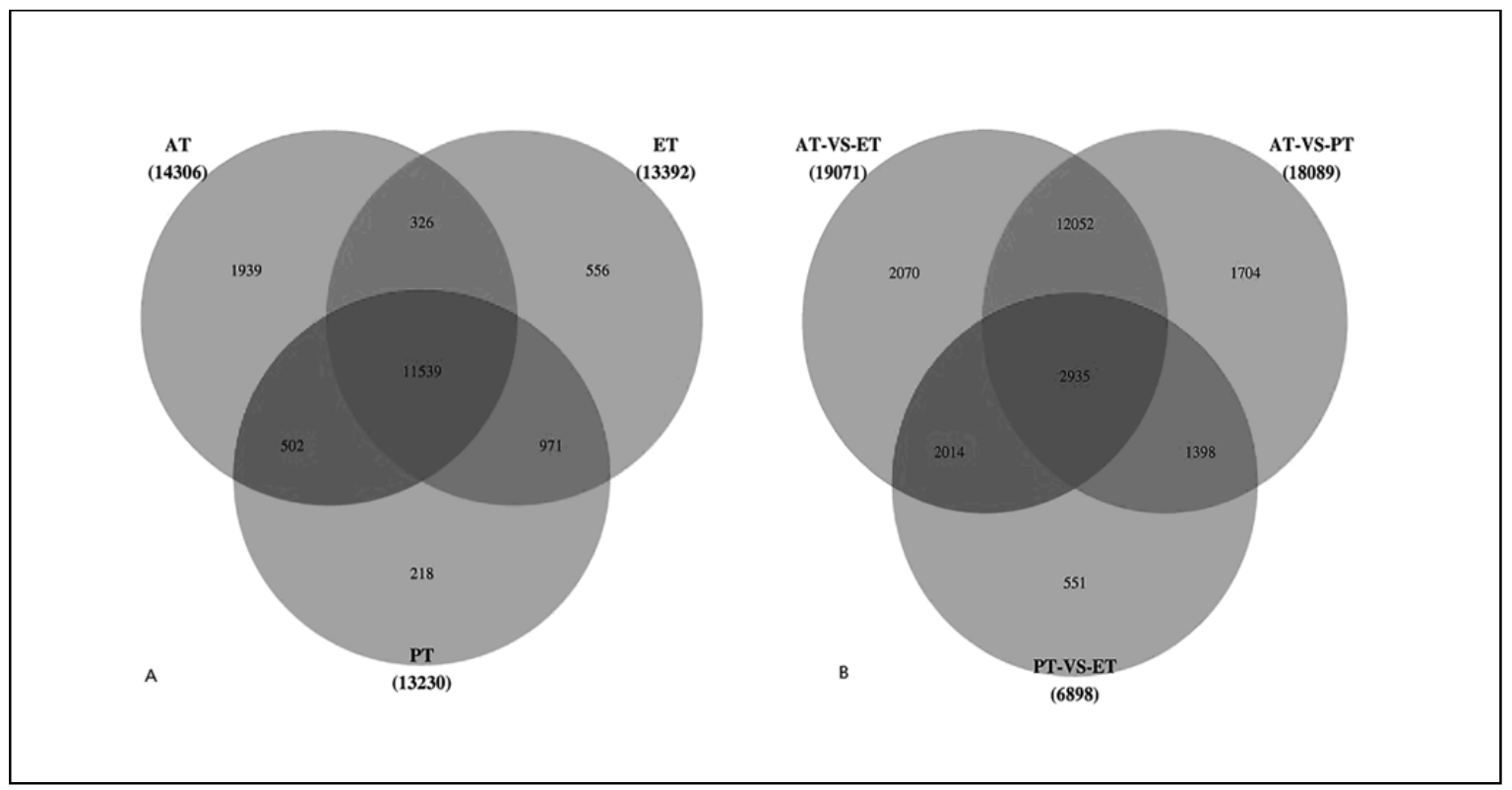

[A: Venn diagram showing annotated genes in AT, PT and ET (average FPKM $>0.5$ ). B: Venn diagram showing differentially expressed genes (DEGs) in AT-VS-PT, PT-VS-ET and AT-VS-ET.]

Fig. 9. Venn diagram of differentially expressed mRNA in the three groups
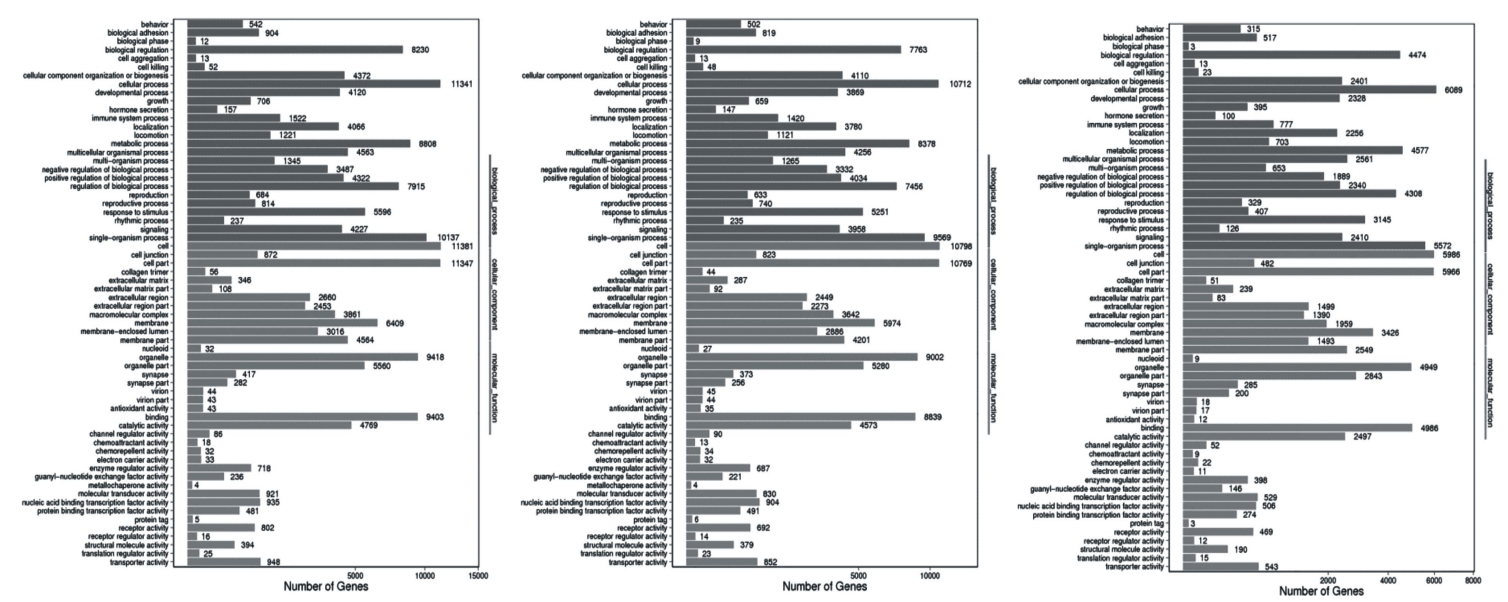

Fig. 10. GO analysis of differentially expressed genes from AT -VS-PT, AT-VS-ET and PT-VS-ET 
Table 3. Functional analysis of the top 20 genes with the highest expression of PT/AT

\begin{tabular}{lccl}
\hline Gene ID & log2 Ratio & Up-Down & \multicolumn{1}{c}{ Function } \\
\hline HMCN1 & 14.02 & Up & cell migration and invasion \\
PLCZ1 & -12.87 & Down & fertilization \\
CDKL3 & -12.97 & Down & cell proliferation \\
C4H7orf34 & -13.07 & Down & $*$ \\
ARMC3 & -13.09 & Down & spermatogenesis \\
ZBBX & & Down & $*$ \\
LOC102174261 & -13.33 & Down & $*$ \\
ASB17 & -13.35 & Down & development of testis0spermatogenesis \\
LOC100860748 & -13.55 & Down & $*$ \\
ZMYND10 & -13.58 & Down & sperm dysmotility0ciliary movement \\
C1H3orf30 & -13.70 & Down & $*$ \\
FSCN3 & -13.90 & Down & sperm development \\
DNHD1 & -13.94 & Down & highly expressed in human brain \\
ROS1 & -14.08 & Down & epididymis \\
FSCB & -14.13 & Down & capacitation of sperm \\
NUP210L & -14.37 & Down & $*$ \\
CAPN11 & -14.38 & Down & germ cell apoptosis \\
DNAH2 & -14.57 & Down & Sperm flagella composition \\
CCDC168 & -14.87 & Down & $*$ \\
TKTL2 & -14.91 & Down & cancer \\
\hline
\end{tabular}

*: No relevant literature was found

Table 4. Functional analysis of the top 20 genes with the highest expression of ET/PT

\begin{tabular}{lccl}
\hline Gene ID & log2 Ratio & Up-Down & Function \\
\hline LOC108637382 & 11.84 & Up & $*$ \\
ROS1 & 10.96 & Up & Cancer \\
LOC102168680 & 10.63 & Up & $*$ \\
LOC102176710 & 10.47 & Up & phosphorylation \\
PPP1R1B & 9.92 & Up & lipid accumulation \\
SLC7A3 & 9.85 & Up & blood capillary \\
LYVE1 & 9.60 & Up & spermatogenesis \\
ERBB3 & 9.35 & Up & $*$ \\
LRRN4CL & 9.33 & Up & anemia \\
ALAS2 & 9.12 & Up & $*$ \\
LOC106502768 & -9.20 & Down & intramuscular fat \\
GLP2R & -9.50 & Down & spermatogenesis \\
RBM15 & -9.61 & Down & $*$ \\
LOC108633254 & -9.64 & Down & satiation \\
CCK & -9.65 & Down & methylation \\
ZNF132 & -10.24 & Down & $*$ \\
LOC102189601 & -10.30 & Down & defense response \\
DEFB119 & -10.62 & Down & Sperm \\
RBM44 & -11.38 & Down & Innate Immunity \\
SMPDL3B & -11.57 & Down &
\end{tabular}

*: No relevant literature was found 

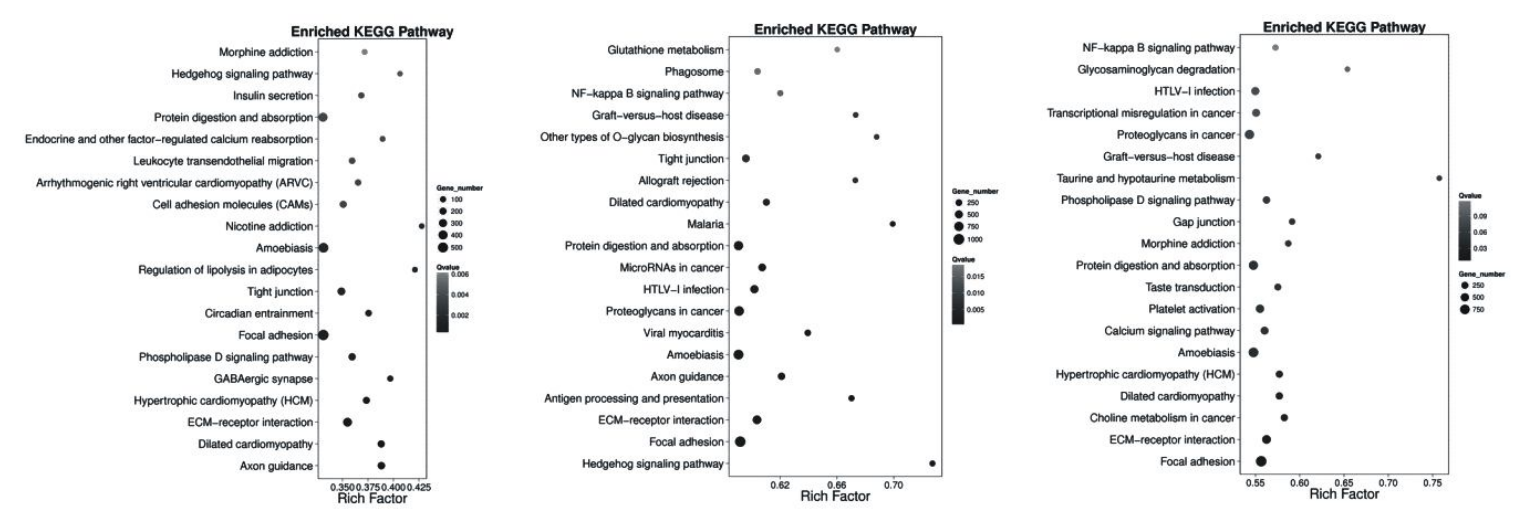

Fig. 11. The top 20 pathways enriched of differently expressed genes (DEGs) from AT, PT and ET goat testes tissues

To better understand the function of differentially expressed genes, the role of differentially expressed genes in spermatogenesis in testicular development in the three age stages of testicular development was verified. We screened the top 20 differentially expressed genes from AT-vs-PT and PT-vs-ET for functional annotation (Tables 3-4). The tables show that the majority of differentially expressed genes in AT-vs-PT are related to sperm maturation and testicular growth, while the majority of differentially expressed genes in PT-vs-ET are related to spermatogenesis and cell differentiation.

KEGG pathways: As shown in Fig. 11, the 20 most enriched pathways were identified by pairwise comparisons of complete DEG lists from every group. Additionally, KEGG analysis revealed several genes involved in MAPK, TGF- $\beta$, mTOR and Wnt signaling. This study focused on the analysis of MAPK, TGF- $B$ and Wnt pathways that influence spermatogenesis.

As stated earlier, the PT and ET libraries of gene expression profiles are similar, so this study only focused on the analysis of PT and AT for the differentially expressed genes in MAPK, TGF- 3 and Wnt signaling pathways in goat testicles. First, in the TGF$B$ (transforming growth factor beta, TGFß) signaling pathway, BAMBI, BMPRI, TGF-ßI, TGF-ßII and SMAD4.6.7 gene expression rose significantly in $\mathrm{PT}$, but lefty, SKP1, DP1, and Smurf1.2 expression decreased (Fig. 12a). Second, in the WNT signaling pathway, the expression levels of P53, FRP, WNT5, Stbm, LRP5 and LRP6 were significantly upregulated. The expression levels of WNT3, GBP, SKP1, B-catenin and WIF1 were significantly upregulated (Fig. 12b). Again, in the MAPK (mitogen-activated protein kinases) 
Indian Journal of Animal Health, June, 2019
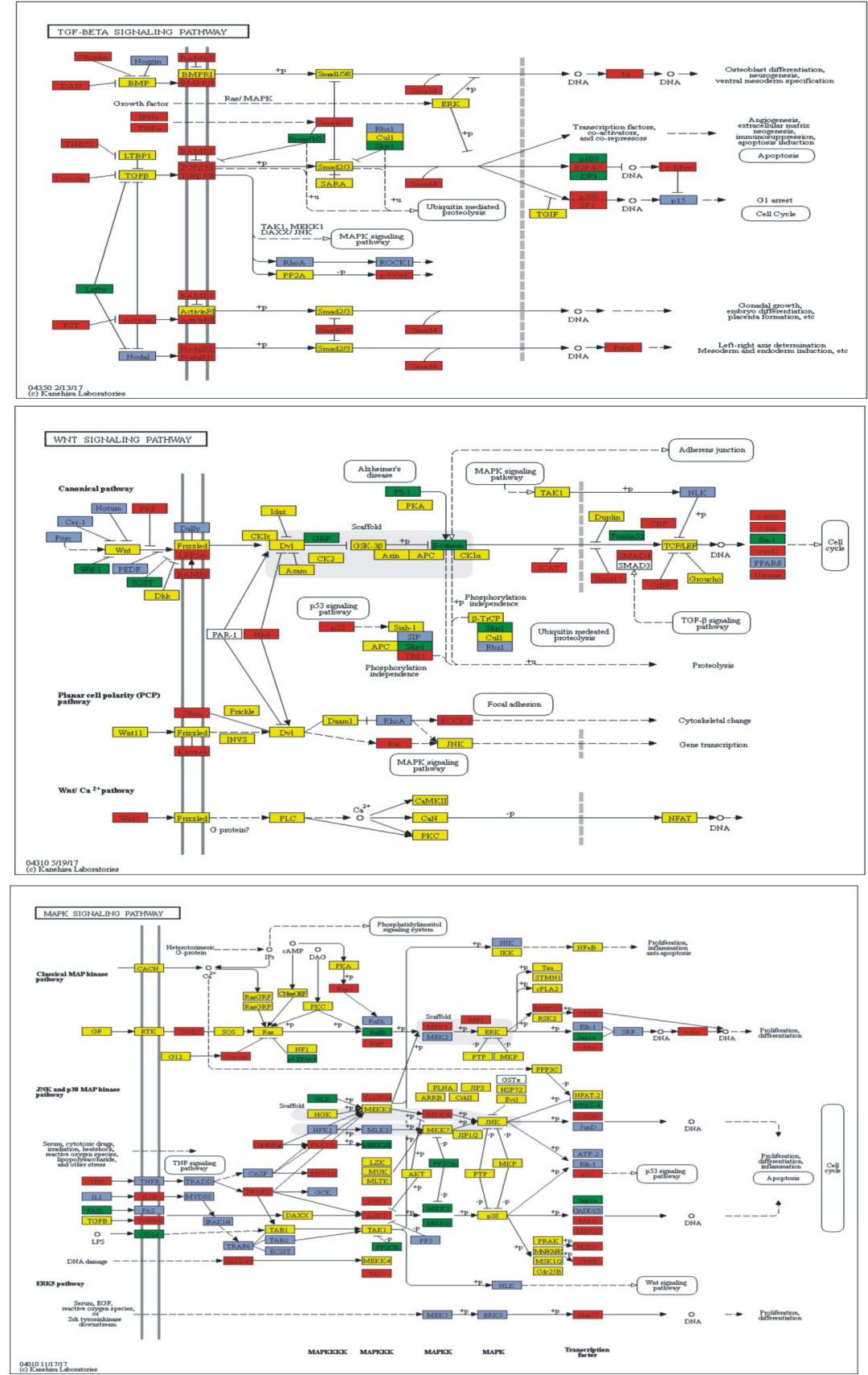

[(a), Wnt (b) and MAPK (c) signaling pathways in goat AT and PT libraries. Red indicates that gene expression is upregulated in PT. Green indicates that gene expression is downregulated. Yellow indicates that gene expression neither downregulated nor upregulated.]

Fig. 12. Localization of differentially expressed genes in TGF- $\beta$, Wnt and MAPK signaling pathways 
signaling pathway, the expression of GRB2, Rap1, Raf1, MEK1, TNF, TGFBR, ASK1 and ASK2 increased significantly, whereas the expression of RafB, FASL, MKK3 and GLK significantly decreased (Fig. 12c).

\section{DISCUSSION}

Testicles are the reproductive organs of male mammals. The basic functions of testicles are to produce sperm and to secrete a variety of sex hormones. Testicular development and spermatogenic stem cell differentiation are the basis of male reproduction and directly affect reproductive health and reproductive performance. Therefore, sequencing the transcriptome of the testicular tissue of goats after birth is helpful to understand the regulatory mechanisms of testicular development. Studying mRNA that influences testicular development and its important role in spermatogenesis can enrich the known gene regulation network during testicular development and spermatogenesis.

In this study, the differentially expressed genes of goat testicles in different development stages were discussed, and genes with significant differences in expression in the three age groups were listed, as shown in Tables 3-4. On one hand, the known key genes can be analyzed and verified: for example, DNAH2, FSCN3, ARMC3 and other genes that are highly expressed during the period of AT and participate in the spermatogenesis process; on the other hand, it can be speculated which new mRNA plays an important role in testicular development and spermatogenesis. This provides the genetic basis and theoretical basis for the study of the biological process of testicular development and spermatogenesis.

As far as we know, this study is the first to use RNA-seq technology on the testes of Dazu Black goats in the infancy, prepuberty, and adult age groups to provide comprehensive and in-depth understanding of the transcriptome profile of testicular tissue. This article comprehensively analyzes a large number of genes associated with spermatogenesis, Sertoli cell proliferation, somatic cell development, and signaling pathways that play an important role in different stages of testis development, which will help deepen the understanding of the regulatory mechanisms of testicular development. For the study of spermatogenesis and other processes and the role of mRNA in testicular development, this study provides effective data.

Analysis of differentially expressed genes: Analyze gene expression levels In this study, we compared the gene expression profiles for goat testis at three developmental stages. We found more differences in adult gene expression profiles. We hypothesized that this is related to sperm production in the testicles of adult goats, more cell types in the testicles and more comprehensive gene expression. Through the analysis of differentially 
expressed genes in different age groups, we found that most of the genes with FPKM values greater than 1,000 in AT were related to spermatogenesis, such as Tnp1, UBB, HMGB4 and HSPB9, and these genes were only highly expressed in AT. It has been reported that the Tnp temporarily replaces the histones in the nucleus during spermatogenesis, and then the Tnp is replaced by the sperm protein. Tnp1 is mainly expressed during late meiosis of male germ cells and is involved in the process of cell deformation during spermatogenesis. The normal expression of Tnp1 is necessary for the correct differentiation of round sperm into mature sperm (Tseden et al., 2007). Catena et al. found HMGB4 in the euchromatin of advanced sperm cells and haploid round sperm cells, suggesting that HMGB4 may play an important role in spermatogenesis, but they did not conduct in-depth studies (Catena et al., 2009). UBB has been proven to play an important role in meiosis (Ryu et al., 2008). We hypothesize that since spermatogenesis involves several meiosis stages, the expression of UBB can be detected even though there is no spermatogenesis in the testicle in the ET and PT stages. HSPB9 is expressed only in the testis (Wit et al., 2004), regulates spermatogenesis and is increased during high temperature (Xun et al., 2015). Therefore, we speculated that the high expression might be related to the spermatogenesis during this period and the collection of samples in hot weather. However, there are no reports on the genetic functions of the highly expressed genes FAM229B, LOC100860923, LOC 102189079 , etc. Although it can be speculated according to expression trends that the functions may be related to spermiogenesis, this must be proven by subsequent experiments. In addition, we also analyzed the genes with high expression in ET, and studies have shown that ribosomal proteins can affect cell proliferation and apoptosis (Sen et al., 2013); there have also been a large number of studies on the role of ribosomal proteins in the reproductive development of aquatic organisms, but no similar articles have been reported in mammals. COCIA 1 and COC1A2 were only highly expressed in ET, possibly because their expressions were related to type A spermatogonial cells, which may mediate the separation and migration of germ cells during the process of spermatogenesis (He et al., 2005).

Analysis of specifically expressed genes in testis - The testicular parenchyma is mainly composed of the seminiferous tubule and testicular stroma. The seminiferous tubules are composed of the boundary membrane and the spermatogenic epithelium, while the spermatogenic epithelium consists of Sertoli cells and germ cells. Mature goat testicles contain spermatogonial cells, sperm cells and other reproductive cells. Among them, spermatogenic cells, Sertoli cells and Leydig cells are testicle-specific cells. In this study, we analyzed the changes of some cell markers in goat testis at different developmental stages. At the ages 
of 1 day and 2 months after birth, the proportion of spermatogonial stem cells in goat testicles was higher, and the expression of related genes including IITGA6, ITGB1, CD34 and Gfra1 was also increased. When spermatogenesis is initiated, other types of spermatogenic cells proliferate and the proportion of spermatogonial stem cells decreases. Therefore, the expression of these markers of spermatogonial stem cells is significantly down regulated in adulthood. In testes of 1 day of age, the AMH (anti-Mullerian hormone, AMH) expression level is much higher than in testes aged 1.5 months and 9 months. This may be related to the fact that AMH is only produced in immature support cells in males and that spermatogonial cells proliferate in large numbers while Sertoli cells mature continuously with testicular growth and development. It has been reported in the literature that the expression of AMH was not detected in adult horses (Ball et al., 2008); however, this study found that it was still expressed in adult testis, although the expression level was very low $(\mathrm{FPKM}<1)$, and there was no statistical significance. We speculated that this difference might be related to species. THBS2 is a marker of embryonic mesenchymal cells, and it has been reported in the literature that it is not expressed in mature mesenchymal cells (Zhang et al., 2004), but its expression was detected in testis of 2 months and 9 months in this experiment.

As seen above, the expression level of most cell markers decreased at 2 and 9 months of age. This paper suggests that after spermatogenesis is initiated, the proportion of different cell types in the testis is changed, the proliferation of spermatogenic cells leads to a significant decrease in the number of spermatogonial stem cells, and thus the gene expression level of spermatogonial stem cells is down regulated. In addition, the maturation of Sertoli cells is the main reason for the decrease of AMH expression.

Analysis of differentially expressed genes - According to analysis of the selected genes in tables 3 and 4 , we found that the results showed that most of the genes differentially expressed in AT-vs-PT were related to the differentiation of sperm cells, such as ARMC3, FSCN3, DNAH2, ASB17, etc. There are also genes associated with cell migration and fertilization, which may be associated with spermatogenesis and sperm movement from the seminiferous tubule to the epididymis, and this involves the testicle secreting certain proteins in preparation for fertilization. Most of the genes differentially expressed in PT-vs-ET were related to spermatogenesis and testicular development, and there are also genes, such as RBM15, that are involved in spermatogenesis: RBM15 is involved in meiosis, and the testicles of 2-month-old goats manifested an ongoing meiosis process.

Analysis of cellular signaling pathways: In the testis, the MAPK signaling pathway is involved in the cell cycle, spermatogenic 
cell differentiation and apoptosis, dynamic changes of the blood-testosterone barrier and adhesion between Sertoli cells and spermatogenic cells (Li et al., 2009). Studies of rat testes have shown that genes involved in MAPK signaling in spermatogenic cells are already actively transcribing genes in the testes of newborn mice (Gnessi et al., 1995; Basciani et al., 2010). In this study, the transcriptional activity of the MAPK signaling pathway was decreased in the mature testis, which may be related to the increase of testosterone level in adulthood.

The Wnt signaling pathway is complex in postnatal goat development. Studies have shown that the Wnt signaling pathway plays a regulatory role in the self-renewal and differentiation of spermatogonial stem cells. Regulating the differentiation and maturation of Sertoli cells through the Wnt

\section{REFERENCES}

Altschul SF, Gish W, Miller W, Myers EW and Lipman DJ, 1990. Blast (basic local alignment search tool). J Mol Biol, 215(3);403-410

Archana P, Katiyar RS, Sharma DN, Farooqui MM and Prakash A, 2014. Postnatal development of testis in Gaddi goat (Capra hircus). Int J Morphol, 32: 166176

Ball BA, Conley AJ, Grundy SA, Sabeur K and Liu IKM, 2008. Expression of antimüllerian hormone $(\mathrm{amh})$ in the equine testis. Theriogenology, 69(5): 624-631 signaling pathway can affect the selfrenewal and differentiation of spermatogonial stem cells and thus participate in the regulation of spermatogenesis (Tanwar et al., 2010). In this experiment, it was found that the transcriptional activity of the Wnt signaling pathway decreased in adulthood, which may be attributed to the fact that the number of Sertoli cells in the goat testis was relatively stable as the adult matured, and the proliferation and differentiation of spermatogonial stem cells entered a relatively stable equilibrium state with the formation of other types of spermatogenic cells.

Conflict of interest: There is no conflict of interest that could be perceived as prejudicing the impartiality of the research reported.

Barcelos B, Fuentes-Soriano S, Watts J, Williams F, Ribeiro FRB et al., 2016. P3046 gene expression in developing goat testes: Sequencing, assembly and identification of caprine spermatogenesis transcriptome. J Anim Sci, 94: 75

Basciani S, Mariani S, Spera G and Gnessi L, 2010. Role of platelet-derived growth factors in the testis. Endocr Rev, 31(6): 916

Buchfink B, Xie C and Huson DH, 2015. Fast and sensitive protein alignment using DIAMOND. Nat Methods, 12: 59-60 
Catena R, Escoffier E, Caron C, Khochbin S and Martianov I et al., 2009. Hmgb4, a novel member of the hmgb family, is preferentially expressed in the mouse testis and localizes to the basal pole of elongating spermatids. Biol Reprod, 80(2): 358-366

Conesa A, Gotz S, Garcia-Gomez JM, Terol J, Talon M et al., 2005. Blast2GO: A universal tool for annotation, visualization and analysis in functional genomics research. Bioinformatics, 21: 3674-3676

Connolly MA, Clausen PA and Lazar JG, 2006. Purification of RNA from animal cells using trizol. CSH Protoc, 2006, DOI:10.1101/pdb.prot4104

De Rooij DG, 2001. Proliferation and differentiation of spermatogonial stem cells. Reproduction, 121(3): 347-354

Dere E, Spade DJ, Hall SJ, Altemus A, Smith J et al., 2017. Identification of sperm mRNA biomarkers associated with testis injury during preclinical testing of pharmaceutical compounds. Toxicol Appl Pharmacol, 320: 1-7

Faucette AN, Maher VA, Gutierrez MA, Jucker JM, Yates DC et al., 2014. Temporal changes in histomorphology and gene expression in goat testes during postnatal development. J Anim Sci, 92: 4440-4448

Guan Y, Liang G, Martin GB and Guan LL, 2017. Functional changes in mRNA expression and alternative pre-mRNA splicing associated with the effects of nutrition on apoptosis and spermatogenesis in the adult testis. Bmc Genomics, 18: 64

Gnessi L, Emidi A, Jannini EA, Carosa E,
Maroder M et al., 1995. Testicular development involves the spatiotemporal control of pdgfs and pdgf receptors gene expression and action. J Cell Biol, 131(4): 1105-1121

He Z, Feng L, Zhang X, Geng Y, Parodi DA et $a l ., 2005$. Expression of col1a1, colla2 and procollagen 1 in germ cells of immature and adult mouse testis. Reproduction, 130(3): 333-341

Jin H, Huang Y, Jin G, Xue Y, Qin X et al., 2015. The vitamin $D$ receptor localization and mRNA expression in ram testis and epididymis. Anim Reprod Sci, 153: 2938

Kim D, Langmead B and Salzberg SL, 2015. HISAT: A fast spliced aligner with low memory requirements. Nat Methods, 12: 357-360

Langmead B and Salzberg SL, 2012. Fast gapped-read alignment with Bowtie 2. Nat Methods, 9: 357-359

Lejeune H, Habert R, Saez JM.(1998). Origin, proliferation and differentiation of Leydig cells. J Mol Endocrinol. 20:1-25

Li MW, Mruk DD and Cheng CY, 2009. Mitogen-activated protein kinases in male reproductive function. Trends Mol Med, 15(4): $159-168$

Pertea M, Pertea GM, Antonescu CM, Chang TC, Mendell JT et al., 2015. String Tie enables improved reconstruction of a transcriptome from RNA-seq reads. Nat Biotechnol, 33: 290-295

Ryu KY, Sinnar SA, Reinholdt LG, Vaccari S, Hall S et al., 2008. The mouse polyubiquitin gene ubb is essential for 
meiotic progression. Mol Cell Biol, 28(3): 1136-1146

Saitou M and Yamaji M, 2012. Primordial germ cells in mice. Csh Perspect Biol, 4: 59-66

Samir H, Nagaoka K, Karen A, Ahmed E, Sayed ME et al., 2015. Investigation the mRNA expression of KISS 1 and localization of kisspeptin in the testes of Shiba goats and its relationship with the puberty and steriodogenic enzymes. Small Ruminant Res, 133: 1-6

Sharma A, Shah SM, Saini N, Kaushik R, Singh MK et al., 2016. Derivation, enrichment and characterization of goat (Capra hircus) spermatogonial stem cells from pre-pubertal testes. Indian J Anim Res, 50(5): 662-667

Sen N, Paul BD, Gadalla MM, Mustafa AK, Sen $\mathrm{T}$ et al., 2012. Hydrogen sulfidelinked sulfhydration of nf-kb mediates its antiapoptotic actions. Mol Cell, 45(1): 13-24

Szatkowska I, Jedrzejczak-Silicka M, Dybus A, Wiszniewska B, Udala J et al., 2017. Expression profile of the SOX9 gene in the testes of sexually immature and mature male goats (Capra hircus), and its potential influence on postnatal testis development. Veterinární Medicína, 62: 253-260

Tanwar PS, Kanekotarui T, Zhang L, Rani P, Taketo MM et al., 2010. Constitutive wnt/ beta-catenin signaling in murine sertoli cells disrupts their differentiation and ability to support spermatogenesis. Biol Reprod, 82(2): 422
Tseden K, Topaloglu O, Meinhardt A, Dev A, Adham I et al., 2007. Premature translation of transition protein $2 \mathrm{mRNA}$ causes sperm abnormalities and male infertility. Mol Reprod Dev, 74(3): 273279

Wit NJWD, Verschuure P, Guido K, King, SM, Jong WWD et al., 2004. Testis-specific human small heat shock protein hspb9 is a cancer/testis antigen, and potentially interacts with the dynein subunit tctel1. Eur J Cell Biol, 83(7): 337-345

Xun, W, Shi L, Cao T, Zhao C and Zhou H, 2015. Dual functions in response to heat stress and spermatogenesis: characterization of expression profile of small heat shock proteins 9 and 10 in goat testis. Biomed Res Int, 2015(5): 1-8, doi: $10.1155 / 2015 / 686239$

Zhang F, Pakarainen T, Zhu F, Poutanen M and Huhtaniemi I. 2004. Molecular characterization of postnatal development of testicular steroidogenesis in luteinizing hormone receptor knockout mice. Endocrinology, 145(3): 1453-1463

Zhao ZQ, Wang LJ, Sun XW, Zhang JJ, Zhao YJ et al., 2015. Transcriptome analysis of the Capra hircus ovary. Plos One, 10: e0121586

Zhu H, Liu C, Li M, Sun J, Song W et al., 2013. Optimization of the conditions of isolation and culture of dairy goat male germline stem cells (mGSC). Anim Reprod Sci, 137: 45-52 